\title{
An assessment of the use of an advanced neural network model with five different training strategies for the preparation of landslide susceptibility maps.
}

\begin{abstract}
Data collection for landslide susceptibility modelling is often an almost inhibitive activity. This has been the reason for quite sometimes landslide was described and modelled on the basis of spatially distributed values of landslide related attributes. This paper presents landslide susceptibility analysis at Selangor area, Malaysia, using artificial neural network model with the aid of remote sensing data and geographic information system (GIS) tools. To meet the objectives, landslide locations were identified in the study area from interpretation of aerial photographs and supported with extensive field surveys. Then, the landslide inventory was grouped into two categories: (1) training data (2) testing data. Further, topographical, geological data and satellite images were collected, processed, and constructed into a spatial database using GIS tools and image processing techniques. Nine landslide occurrence attributes were selected and analyzed using an artificial neural network model to generate the landslide susceptibility maps. Landslide location data (training data) were used for training the neural network and five training sites were selected randomly in this case. The use of five training sites ensemble to investigate the model reliability, including the role of the thematic variables used to construct the model, and the model sensitivity to changes in the selection of the training sites. By studying the variation of the neural network's susceptibility estimate, the error associated with the model is determined. The results of the neural network analysis are shown on five sets of landslide susceptibility maps. Then the susceptibility maps were validated using "receiver operating characteristics (ROC)" method as a measure for the model verification. Landslide training data which were not used during the training of the neural network was used for the verification of the maps. The results of the analysis were verified using the landslide location data and compared between five different cases. Qualitatively, the model seems to give reasonable results with accuracy observed was $87 \%, 83 \%, 85 \%, 86 \%$ and $82 \%$ for five different training sites respectively.
\end{abstract}

Keyword: ANN; Fuzzy; Landslide; Susceptibility; GIS; Remote sensing. 